\title{
Observation of Quantum Jumps in a Single Atom
}

\author{
J. C. Bergquist, Randall G. Hulet, Wayne M. Itano, and D. J. Wineland \\ Time and Frequency Division, National Bureau of Standards, Boulder, Colorado 80303
}

(Received 23 June 1986)

\begin{abstract}
We detect the radiatively driven electric quadrupole transition to the metastable ${ }^{2} D_{5 / 2}$ state in a single, laser-cooled $\mathrm{Hg}$ II ion by monitoring the abrupt cessation of the fluorescence signal from the laser-excited ${ }^{2} S_{1 / 2} \rightarrow{ }^{2} P_{1 / 2}$ first resonance line. When the ion "jumps" back from the metastable $D$ state to the ground $S$ state, the $S \rightarrow P$ resonance fluorescence signal immediately returns. The statistical properties of the quantum jumps are investigated; for example, photon antibunching in the emission from the $D$ state is observed with $100 \%$ efficiency.
\end{abstract}

PACS numbers: $32.80 . P \mathrm{j}, 42.50 . \mathrm{Dv}$

Recently, a few laboratories have trapped and radiatively cooled single atoms ${ }^{1-4}$ enabling a number of unique experiments to be performed. One of the experiments now possible is to observe the "quantum jumps" to and from a metastable state in a single atom by monitoring of the resonance fluorescence of a strong transition in which at least one of the states is coupled to the metastable state. When the atomic electron moves to the metastable state, the fluorescence from the strongly driven transition disappears. When the electron drops back into the strongly driven transition, the fluorescence abruptly returns. Thus the strong transition fluorescence is a monitor of the quantum state of the atom. Several years ago, Dehmelt had proposed this optical double-resonance scheme (terming it electron shelving) as an amplification mechanism to detect a weak transition in singleatom spectroscopy. ${ }^{5}$ This technique has been used for several years ${ }^{6}$ in high-resolution spectroscopic studies of samples of many laser-cooled ions, achieving quantum amplifications of $10^{6}$ and higher. In 1981, electron-shelving amplification was used to perform optical-optical double resonance in a single, lasercooled, trapped ion. ${ }^{2}$ While the signal-to-noise ratio in that experiment was not sufficient to see quantum jumps directly, the fact that the atomic fluorescence would be bistable was noted. More recently, the statistics of quantum switching in a single atom have been theoretically treated in some detail first by Cook and $\mathrm{Kimble}^{7}$ and subsequently by several other authors. ${ }^{8-13}$ In this Letter we report the clear experimental demonstration of quantum jumps in a single laser-cooled ${ }^{198} \mathrm{Hg}^{+}$ion stored in a miniature radio-frequency trap.

The basic idea for quantum switching and the associated statistics is illustrated with the three-level system shown in Fig. 1 for the $\mathrm{Hg} \mathrm{II}$ ion. In $\mathrm{Hg}$ II there is a "strong" resonance transition from the $5 d^{10} 6 s^{2} S_{1 / 2}$ ground state to the $5 d^{10} 6 p^{2} P_{1 / 2}$ state near $194 \mathrm{~nm}$. The lifetime of the ${ }^{2} P_{1 / 2}$ state has been measured elsewhere to be $2.3 \pm 0.3$ ns. $^{14}$ Additionally, there is a "weak" electric quadrupole transition from the ${ }^{2} S_{1 / 2}$ ground state to the $5 d^{9} 6 s^{22} D_{5 / 2}$ state near $281.5 \mathrm{~nm}$. The lifetime of the metastable $D$ state has recently been measured to be about $0.1 \mathrm{~s} .{ }^{15,16}$ A laser tuned just below resonance on the highly allowed $S-P$ transition will cool the ion and, in our case, scatter up to $5 \times 10^{7}$ photons/s. By collecting even a small fraction of the scattered photons, we can easily monitor the quantum state of the atom. If only the strong transition is radiatively driven, then for averaging times long compared to the $P_{1 / 2}$ state lifetime and mean $S \rightarrow P$ excitation time, a steady fluorescence level is expeced, corresponding to the atom rapidly cycling between the $S$ and $P$ state. If radiation to drive the weak transition is also admitted, then the atom will occasionally be driven into the metastable state and the fluorescence from the strong transition will abruptly disappear. The cessation of the scattering of many photons on the strong transition for one photon absorbed on the weak transition permits unit detection efficiency of the transition to the metastable state. ${ }^{6,17}$ Some time later, the atom returns spontaneously or is driven out of the $D$ state back to the ground state, which causes a sudden return of the fluorescence on the strong transition. The random on/off "telegraphic" signal provides a direct indication of the quantum state of the ion.

The experimental setup is largely the same as that

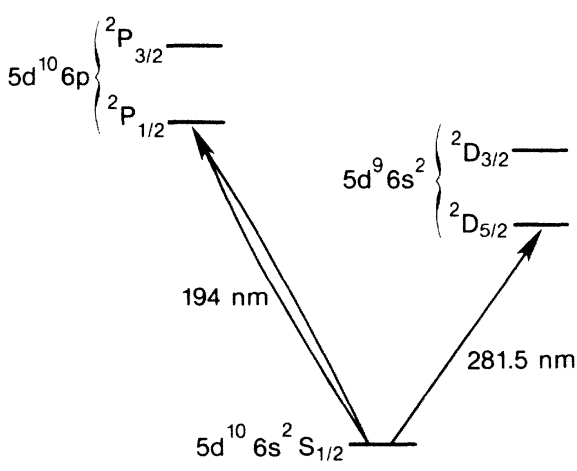

FIG. 1. Simplified optical energy-level diagram for $\mathrm{Hg} \mathrm{II}$. 
used in the two-photon spectroscopic study of the ${ }^{2} S_{1 / 2^{-}}{ }^{2} D_{5 / 2}$ transition in $\mathrm{Hg}$ II reported earlier. ${ }^{15}$ In the present experiment, one ion (on some occasions, two ions) was loaded ${ }^{2}$ into the trap and cooled to below 25 $\mathrm{mK}$ by $2-5 \mu \mathrm{W}$ of sum-frequency-generated radiation near $194 \mathrm{~nm}$ (spot size $w_{0} \approx 10 \mu \mathrm{m}$ ) tuned just below the ${ }^{2} S_{1 / 2^{-}}{ }^{2} P_{1 / 2}$ first resonance transition. The fluorescence light scattered by the ions was detected at right angles to the 194-nm beam with an overall detection efficiency of about $5 \times 10^{-4}$. Peak signal counts exceeded $2 \times 10^{4} / \mathrm{s}$ against a background counting rate of $200 / \mathrm{s}$. This high counting rate permitted us to monitor the fluorescence at a $10-\mathrm{ms}$ sampling rate with a reasonable signal-to-noise ratio. The fast sampling rate is necessary because of the $100-\mathrm{ms}$ lifetime of the ${ }^{2} D_{5 / 2}$ state.

Radiation from a frequency-stabilized ring dye laser near ${ }^{15} 563 \mathrm{~nm}$ was doubled to $281.5 \mathrm{~nm}$ in order to drive the ${ }^{2} S_{1 / 2^{-2}} D_{5 / 2}$ electric-quadrupole-allowed transition directly. ${ }^{18}$ The power of the $281.5-\mathrm{nm}$ radiation could be adjusted to as much as $20 \mu \mathrm{W}$. The beam was focused at the center of the trap to a spot size $w_{0}$ of approximately $25 \mu \mathrm{m}$. A magnetic field of approximately $1 \mathrm{mT}(10 \mathrm{G})$ was applied parallel to the electric field vector of the $281.5-\mathrm{nm}$ radiation and perpendicular to its direction of propagation. The selection rule for the electric-quadrupole-allowed transitions to the various Zeeman states for this configuration is $\Delta m_{J}=1$. The frequency of the $281.5-\mathrm{nm}$ radiation was tuned to resonance with the ${ }^{2} S_{1 / 2} \rightarrow{ }^{2} D_{5 / 2} \quad\left(m_{J}\right.$ $=-\frac{1}{2} \rightarrow m_{J}=+\frac{1}{2}$ ) Zeeman component for the quantum switching results reported here. Also, the resonance signal obtained from the $P_{1 / 2}$ fluorescence counts during scanning of the 281.5 -nm laser over this component revealed a linewidth of less than $8 \mathrm{MHz}$. If this width is due to Doppler broadening, the ion temperature (in the pseudopotential well) is less than 25 $\mathrm{mK}$ and the ion is estimated to be confined to a volume characterized by a linear dimension of less than $0.25 \mu \mathrm{m}$.

In order to observe the quantum jumps, we monitor the strong fluorescence at $194 \mathrm{~nm}$ while simultaneously admitting the $281.5-\mathrm{nm}$ radiation. A computer strobes and displays the detected 194-nm fluorescence counts accumulated in a counter at $10-\mathrm{ms}$ intervals for running times of $40 \mathrm{~s}$. These data are then stored and the process repeated, but now without the $281.5-\mathrm{nm}$ radiation, or, in some cases, with the $281.5-\mathrm{nm}$ laser detuned from resonance. We repeat the entire sequence numerous times for different power levels of the $281.5-\mathrm{nm}$ light and for different detunings of the 194-nm radiation. An example of $4 \mathrm{~s}$ of a sequential set of data with the $281.5-\mathrm{nm}$ radiation $(0.3 \mu \mathrm{W})$ first off and then on is shown in Figs. 2(a) and 2(b), respectively.

For the general case of coherent excitation, it is

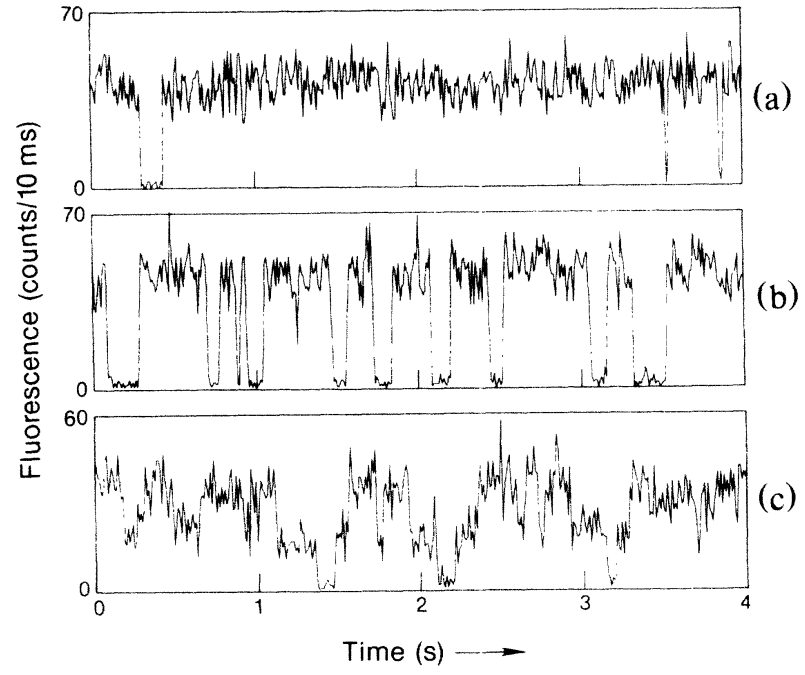

FIG. 2. Samples of the quantum-jump data. (a) The fluorescence counts detected with no resonance radiation exciting the weak $S_{1 / 2}-D_{5 / 2}$ transition. The few jumps observed are likely due to collisions with background $\mathrm{Hg}$ atoms and radiative decay to the $D_{3 / 2}$ state. (b) Both the 194- and 281.5-nm radiation are applied simultaneously. Compared to (a), the interruptions to the fluorescence signal are more frequent. (c) Two ions are trapped and cooled. The interruptions to the fluorescence signal show two levels corresponding to loss of fluorescence from one or both ions. The sampling rate for all the data is $10 \mathrm{~ms}$ per point; the length of each sample is $4 \mathrm{~s}$.

necessary to examine the off-diagonal terms of the density matrix in order to include possible coherence effects. ${ }^{8,13}$ However, for the conditions of our experiment, that is, for times longer than the inverse of the excitation and spontaneous emission rates on the strong transition, and when the excitation and emission rates on the strong transition exceed those on the weak transition, the dynamics of the quantum-jump process can be described by effective two-state rate equations for coherent or incoherent excitation. ${ }^{8}$ As a consequence there exist simple probabilities per unit time $R_{+}$and $R_{-}$that the electron makes an upward or downward jump on the weak transition. Using the theory of Ref. 8 adapted to arbitrary detuning, and with the additional condition relevant to our experiment that the radiation driving the strong transition is well below saturation, we find that the Einstein $A$ coefficient for the spontaneous decay of the weak transition is related to these probabilities by

$$
A\left({ }^{2} D_{5 / 2}\right) \approx R_{-}-R_{+} \cdot
$$

From data similar to those of Fig. 2(b), we plotted the distribution of off times $\tau_{\text {off }}$ and the distribution of on times $\tau_{\text {on }}$. Theory ${ }^{7,8}$ predicts that the probability density for the time duration of the off (and on) intervals 
is given by

$$
W_{\text {off }}\left(\tau_{\text {off }}\right)=R_{-} \exp \left(-R_{-} \tau_{\text {off }}\right)
$$

for time off, and by

$$
W_{\text {on }}\left(\tau_{\text {on }}\right)=R_{+} \exp \left(-R_{+} \tau_{\text {on }}\right)
$$

for time on. Thus, $R_{+}$and $R_{-}$are found from exponential least-squares fits to the data, and from these and Eq. (1) we determine the $A$ coefficient for the metastable ${ }^{2} D_{2 / 5}$ state. Unfortunately, the data analysis is complicated by the background events as indicated in Fig. 2(a). Although we have not unambiguously determined their origin, two contributions are collisions with background mercury atoms which temperorarily heat the ions ${ }^{2}$ and radiative decay from the ${ }^{2} P_{1 / 2}$ state to the ${ }^{2} D_{3 / 2}$ state. The lifetime of the ${ }^{2} D_{3 / 2}$ state has recently been measured to be about $20 \mathrm{~ms}$. According to theory ${ }^{19}$ it decays with nearly equal probability to the lower-lying ${ }^{2} D_{5 / 2}$ state and to the ground state. We estimate ${ }^{17}$ the probability that a mercury ion in the ${ }^{2} P_{1 / 2}$ state will decay to the ${ }^{2} D_{3 / 2}$ state as $3 \times 10^{-7}$. In the present experiment there is no direct measure of the background pressure at the trap, but estimates based on ion-pump current are consistent with the observed frequency of background events. Estimated recooling rates are also consistent with the data.

If the background interruptions in the fluorescence signal were due solely to collisions, one would expect their rate of occurrence to be independent of the 194$\mathrm{nm}$ scattering rate. The average duration of the off times after a collision would be a function of the 194$\mathrm{nm}$ intensity and detuning, since these parameters would affect the recooling rate. If the background interruptions were due solely to radiative decay, one would expect their rate of occurrence to be proportional to the 194-nm scattering rate, but their average duration to be fixed, since this would depend only on the metastable decay rates. The present data indicate that both processes may be present in that both the rate and the average duration of off times vary with scattering rate. Even with these background events, we find the lifetime for spontaneous emission from the ${ }^{2} D_{5 / 2}$ level given by Eq. (1) to be $90 \pm 30 \mathrm{~ms}$, which is in agreement with the earlier measurements. ${ }^{15,16}$

Also of interest is the two-time intensity-correlation function ${ }^{7-13}$ for the 194-nm fluorescence:

$$
C(\tau)=\langle I(t) I(t+\tau)\rangle \text {. }
$$

This expression can be written as ${ }^{7}$

$$
C(\tau)=\langle I\rangle^{2}+\left(\left\langle I^{2}\right\rangle-\langle I\rangle^{2}\right) \exp \left[-\left(R_{+}+R_{-}\right) \tau\right],
$$

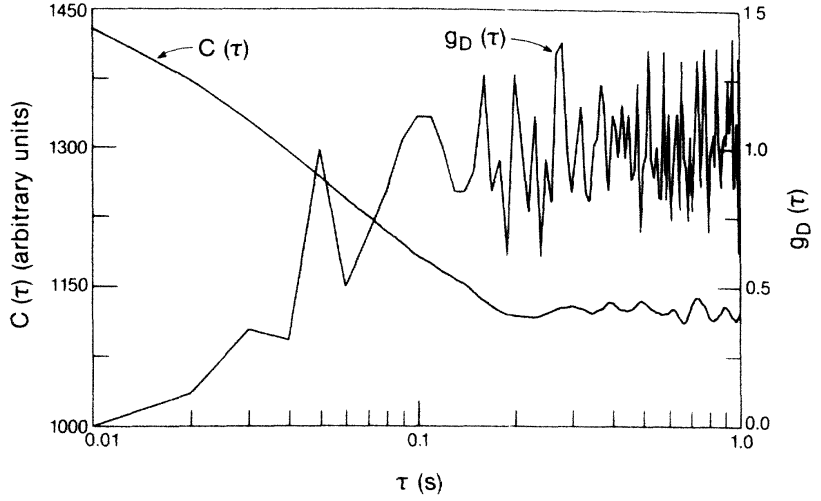

FIG. 3. Plots of the 194-nm fluorescence intensity correlation function $C(\tau)$ and the $281.5-\mathrm{nm}$ emission correlation function $g_{D}(\tau)$. Photon antibunching in the $281.5-\mathrm{nm}$ emission is inferred from the observation that $g_{D}(\tau) \rightarrow 0$ as $\tau \rightarrow 0$.

where

$$
\begin{aligned}
& \langle I\rangle=I_{0} R_{-} /\left(R_{+}+R_{-}\right), \\
& \left\langle I^{2}\right\rangle-\langle I\rangle^{2}=I_{0}^{2} R_{+} R_{-} /\left(R_{+}+R_{-}\right)^{2} .
\end{aligned}
$$

In Fig. 3 we plot the two-time intensity-correlation function for a typical run. The values of $R_{+}$and $R_{-}$ obtained from a least-squares fit agree with those derived from the distributions of off and on times.

Other statistical properties can be derived from the quantum switching data. For example, if one neglects the background interruptions to the fluorescence signal, each upward (downward) transition in the fluorescence can be assumed to mark the emission (absorption) of a ${ }^{2} D_{5 / 2}{ }^{2} S_{1 / 2}$ photon. Let $g_{D}(\tau)$ denote the probability that the (assumed) emission of a $281.5-\mathrm{nm}$ photon is followed by the (assumed) emission of another $281.5-\mathrm{nm}$ photon at a time $\tau$ later, normalized to 1 at $\tau=\infty$. For our experimental conditions, theory predicts that $g_{D}(\tau)=1-\exp \left[-\left(R_{+}+R_{-}\right) \tau\right]$, and this is in agreement with the data. In Fig. 3 we plot $g_{D}(\tau)$ from some of our quantum switching data. The fact that $g_{D}(\tau) \rightarrow 0$ at $\tau=0$ implies the existence of photon antibunching ${ }^{11,13}$ in the $281.5-\mathrm{nm}$ radiation from the ${ }^{2} D_{5 / 2}$ state. Because of the quantum amplification in the $S-P$ scattering loop the photon antibunching is detected with nearly $100 \%$ efficiency.

Finally, in Fig. 2(c) we show quantum switching for the case of two laser-cooled and trapped ions (estimated separation $\approx 2.5 \mu \mathrm{m}$ ). There are three distinct levels of fluorescence corresponding to (a) a maximum when both ions are in the $S-P$ scattering loop, (b) an intermediate level when one ion is shelved in the $D$ state and only one ion is scattering, and (c) no fluorescence in the rare cases when both ions are shelved in the $D$ state. 
In summary, we have demonstrated quantum jumps or switching in a single atom. We have analyzed the statistics and found agreement with earlier published values for the lifetime of the $5 d^{9} 6 s^{22} D_{5 / 2}$ state in $\mathrm{Hg}$ II. It is interesting to speculate about a single atom in which the upper state on the weak transition is extremely long lived and excited by adiabatic rapid passage. ${ }^{20}$ In this case the random nature of the excitation is eliminated and one could realize a single-atom switch or flip flop.

The authors are pleased to acknowledge the expert technical assistance of C. Manney during parts of this experiment, and the help of D. J. Larson in the earlier work to obtain single-ion cooling. We are appreciative to H. J. Kimble for helpful discussions of "quantum jumps" and to J. J. Bollinger, R. E. Drullinger, and L. Hollberg for comments on the manuscript. We gratefully acknowledge the support of the U.S. Air Force Office of Scientific Research and the U.S. Office of Naval Research. We note that a Letter by Nagourney, Sandberg, and Dehmelt ${ }^{21}$ describing quantum jumps in a single $\mathrm{Ba}^{+}$atom has appeared subsequent to the submission of our Letter.

1W. Neuhauser, M. Hohenstatt, P. Toschek, and H. Dehmelt, Phys. Rev. A 22, 1137 (1980).

${ }^{2}$ D. J. Wineland and W. M. Itano, Phys. Lett. 82A, 75 (1981).

${ }^{3}$ W. Nagourney, G. Janik, and H. Dehmelt, Proc. Natl. Acad. Sci. U.S.A. 80, 643 (1983).

${ }^{4}$ G. Janik, W. Nagourney, and H. Dehmelt, J. Opt. Soc. Am. B 2, 1251 (1985).

${ }^{5}$ H. G. Dehmelt, Bull. Am. Phys. Soc. 20, 60 (1975), and
J. Phys. (Paris), Colloq. 42, C8-299 (1981).

${ }^{6}$ D. J. Wineland, J. C. Bergquist, W. M. Itano, and R. E. Drullinger, Opt. Lett. 5, 245 (1980); D. J. Wineland, W. M. Itano, J. C. Bergquist, J. J. Bollinger, and J. D. Prestage, in Atomic Physics, edited by R. S. Van Dyck, Jr., and E. N. Fortson (World Scientific, Singapore, 1985), Vol. 9.

${ }^{7}$ R. J. Cook and H. J. Kimble, Phys. Rev. Lett. 54, 1023 (1985)

${ }^{8}$ H. J. Kimble, R. J. Cook, and A. L. Wells, Phys. Rev. A 34, 3190 (1986).

${ }^{9} \mathrm{~T}$. Erber and S. Putterman, Nature 318, 41 (1985).

10J. Javanainen, Phys. Rev. A 33, 2121 (1986).

${ }^{11}$ A. Schenzle, R. G. DeVoe, and R. G. Brewer, Phys. Rev. A 33, 2127 (1986).

${ }^{12}$ C. Cohen-Tannoudji and J. Dalibard, Europhys. Lett. 1, 441 (1986).

${ }^{13}$ D. T. Pegg, R. Loudon, and P. L. Knight, Phys. Rev. A 33, 4085 (1986).

${ }^{14} \mathrm{P}$. Eriksen and O. Poulsen, J. Quant. Spectrosc. Radiat. Transfer 23, 599 (1980).

15 J. C. Bergquist, D. J. Wineland, W. M. Itano, H. Hemmati, H.-U. Daniel, and G. Leuchs, Phys. Rev. Lett. 55, 1567 (1985).

${ }^{16}$ C. E. Johnson, Bull. Am. Phys. Soc. 31, 957 (1986).

${ }^{17}$ D. J. Wineland, W. M. Itano, J. C. Bergquist, and F. L. Walls, in Proceedings of the 35th Annual Symposium on Frequency Control, Philadelphia, 1981 (Electronic Industries Association, Washington, DC, 1981), p. 602.

${ }^{18} \mathrm{D}$. J. Wineland, J. C. Bergquist, R. E. Drullinger, H. Hemmati, W. M. Itano, and F. L. Walls, J. Phys. (Paris), Colloq. 42, C8-307 (1981).

${ }^{19}$ R. H. Garstang, J. Res. Natl. Bur. Stand. Sect. A 68, 61 (1964).

${ }^{20}$ See, for example, A. Abragam, Principles of Nuclear Magnetism (Clarendon, Oxford, 1961), p. 65.

${ }^{21}$ W. Nagourney, J. Sandberg, and H. Dehmelt, Phys. Rev. Lett. 56, 2727 (1986) 